
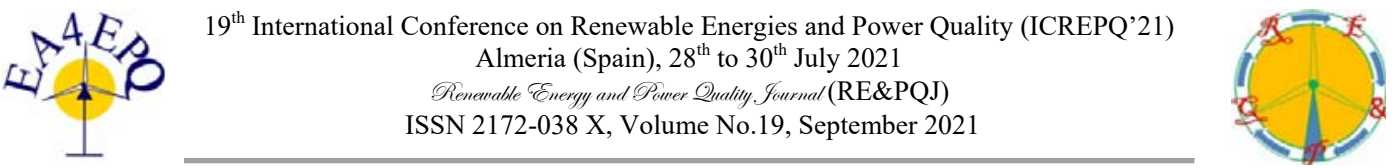

\title{
The Inclusion of Power Gyrator Topologies as Energy Processing Cells in Photovoltaic Solar Conversion
}

\author{
Herminio Martínez-García and Encarna García-Vílchez \\ Department of Electronic Engineering \\ Escola d'Enginyeria de Barcelona Est (EEBE), Technical University of Catalonia (UPC)-BarcelonaTech \\ Campus of Diagonal-Besòs, 08019 Barcelona (Spain) \\ Phone number:+0034.93.413.72.90, e-mail: herminio.martinez@upc.edu, encarna.garcia.vilchez@upc.edu
}

\begin{abstract}
This paper will provide a classification of high efficiency switching power-gyrator structures and their use as cells for energy processing in photovoltaic solar facilities. Having into account the properties of these topologies presented in the article, their inclusion in solar facilities allows increasing the performance of the whole installation.
\end{abstract}

Therefore, the design, simulation and implementation of a Gtype power gyrator are carried out throughout the text. In addition, in order to obtain the maximum power from the photovoltaic solar panel, a maximum power point tracking (MPPT) is mandatory in the energy processing path. Therefore, the practical implementation carried out includes a control loop of the power gyrator in order to track the aforementioned maximum power point of the photovoltaic solar panel.

Key words. Power electronics, DC-DC conversion, power gyrators, maximum power point tracking (MPPT), photovoltaic panels.

\section{Introduction}

The arrival of the switching semiconductor devices in the decade of the $1950 \mathrm{~s}$ carried out the appearance of switching converters and switching power supply systems. The major development of DC-DC converters took place at the beginning of the decade of 1960 s, when switching semiconductors were feasible and affordable devices, being applied in the aerospace industry as one of their first uses. On the other hand, the initial concept of gyrator is referred to networks with certain interesting properties. They are attractive for the synthesis of inductive elements with properties nearer to the ideality than their counterparts of wound core. The concept of gyrator was introduced firstly by Tellegen in his paper 'The gyrator, a new electric network element' published in 1948 [1], in which mention is made for a network with unique properties, and was considered as a new electrical network element added to those already known. The treatment of the subject by Tellegen is rather theoretical and does not venture into the practical design of these elements, although formally founded their behavior and some of its properties. The term 'gyrator' is, since then, used to call this kind of network, of which one of the first was introduced by the same Tellegen for a toroidal ferromagnetic core wound at one end, and separated by a dielectric segment at the other. The introduction of the gyrator circuit concept in power processing (highefficiency switching-mode power gyrators) is due to Singer, who presented a particular gyrator in the circuits named POPI (power output=power input), describing the ideal behavior of a particular switching power converter structure [2]. In 2005 it was shown that the gyrator circuits were unstable, and calculated the stable conditions necessary for its possible implementation [3], [4].

The article is organized as follows: In Section 2 an introduction to gyrator circuits and their classification are carried out; Section 3 explains the use of power gyrators for energy processing in solar energy facilities, together with the use of a MPPT subsystem; Section 4 deals with the design and implementation of a particular power gyrator implementation for the energy processing from a photovoltaic solar panel and its main simulation and experimental results. The article concludes with the main conclusions in Section 5.

\section{Power Gyrator Concept}

The concept of power gyrator introduced in [2] relates to a general sort of circuits named POPI, describing the ideal behavior of a switched-mode power converter. In general two big groups of power gyrators can be found: $G$-type and $R$-type gyrators.

\section{A. G-Type Gyrator}

A power gyrator type $G$ (Figure 1) is defined as a switching converter which satisfies equations (1) and with the characteristic that the input current and output current are not pulsed.

$$
i_{1}=g v_{2} ; i_{2}=g v_{1},
$$

where the parameter $g$ is the conductance of the gyrator. The $G$-type power gyrator with controlled output current behaves like a current source in its output port. 

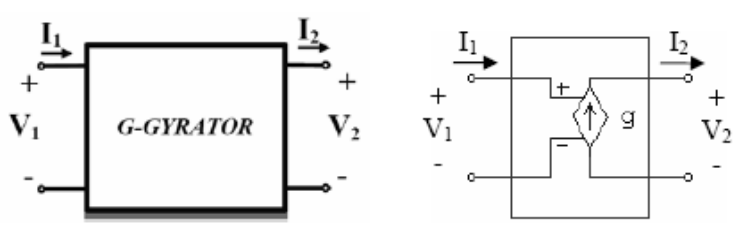

Fig. 1. Basic block of a $G$-type power gyrator.

The general idea of a $G$-type power gyrator is to achieve a controlled dependent current source that depends on the input voltage and a gain factor $g$. If the parameter $g$ is adjustable, a VCCS (voltage-controlled output current source) can be obtained.

The two-port $G$-type power gyrators that can be found are of fourth order, that is, the buck with input filter (BIF), the boost converter with output filter (BOF), the Cuk converter and the Ćuk isolated converter, as illustrated, respectively, in figure 2.

In the present work, a BIF G-type gyrator is used in order to validate the use of power gyrators for energy processing in photovoltaic solar facilities.

\section{B. BIF Gyrator}

The BIF G-type gyrator is a DC/DC switching converter, in particular a buck regulator with input filter. The BIF structure depicted in Figure 2 is an unstable system; therefore, its implementation may not be viable. In [4] it is demonstrated the need of including a damping network to get the system to reach stability and how to calculate it. The proposed circuit, including the proposed stability network, is shown in Figure 3.

The analysis of the BIF G-type gyrator controlled by means of a sliding control loop shows that the system must meet a series of inequalities or conditions to obtain the necessary stability of the circuit; in particular [4], [5]:

$$
\begin{gathered}
R_{d} C_{d}<\frac{C_{1}+C_{d}}{g^{2} R} \\
R_{d} C_{d}>g^{2} R L_{1} \\
g^{2} R R_{d}^{2} C_{d}^{2}+g^{2} R L_{1}\left(C_{1}+C_{d}\right)<\left(g^{4} R^{2} L_{1}+C_{d}\right) R_{d} D_{d}
\end{gathered}
$$

\section{R-Type Gyrator}

A power $R$-type gyrator is defined as a switching converter with a switch topology characterized by (5):

$$
v_{1}=r i_{2} ; v_{2}=r i_{1}
$$

where $r$ is the resistance implemented by the gyrator.

The simpler $R$-type power gyrators are shown in Figure 4. These converters are the boost with output filter (BOF) converter, the Ćuk converter, and Ćuk converter with galvanic isolation.

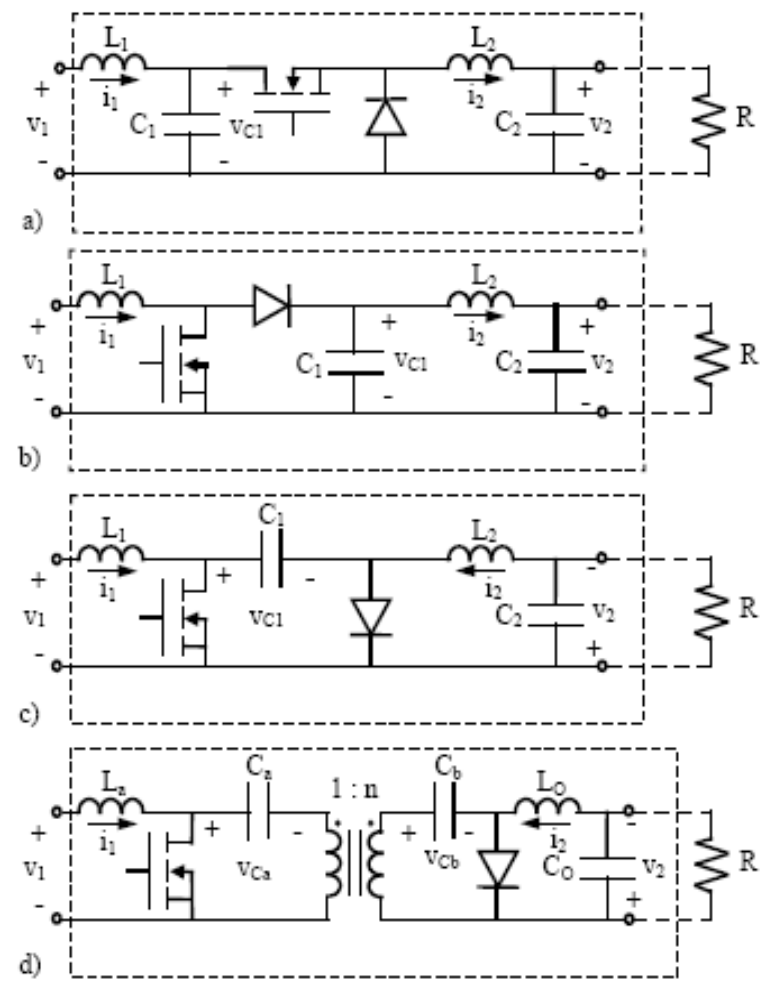

Fig. 2. Classification of $G$-type power gyrators: (a) Buck with input filter (BIF), (b) boost with output filter (BOF), (c) Ćuk converter, and $(d)$ Ćuk isolated.

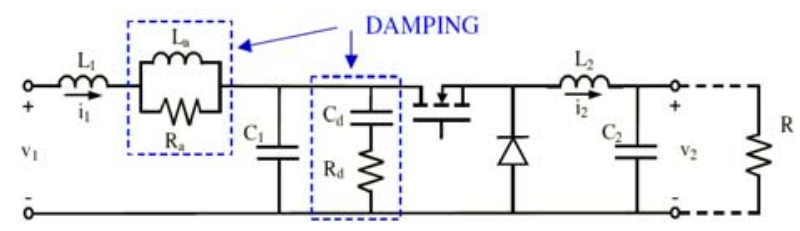

Fig. 3. BIF gyrator with damping network.

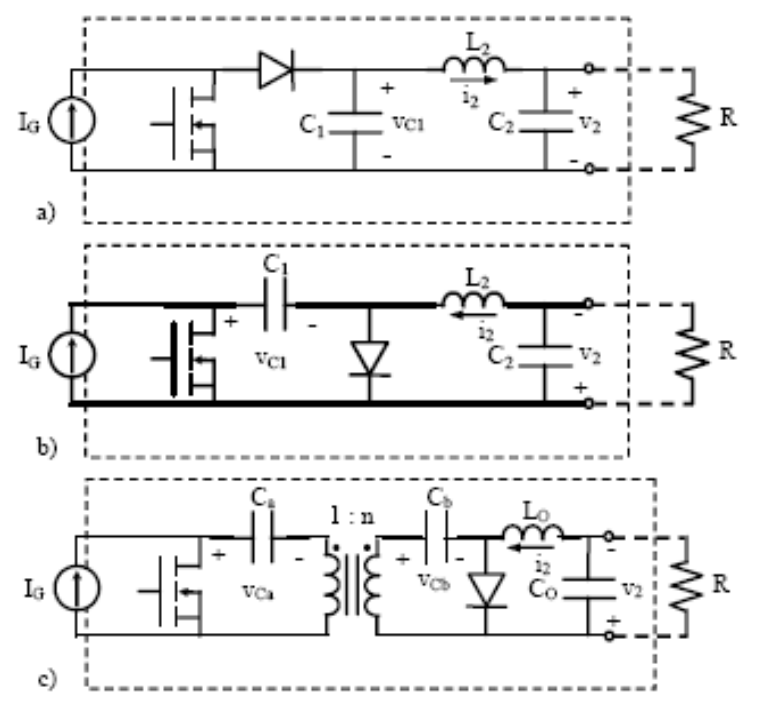

Fig. 4. Classification of $R$-type gyrators: (a) boost with output filter (BOF), (b) Ćuk converter, and (c) Ćuk isolated.

From these different structures, the more used is the first one (the BOF converter). 


\section{Design of a BIF Power Gyrator for the Energy Acquisition of a Photovoltaic Panel}

In this section, in order to demonstrate the feasibility of developing power gyrator structures for solar energy applications, the design and implementation of a BIF Gtype power gyrator are carried out. The objective is to process the energy provided by a photovoltaic panel. The panel used for the implementation of the application is supplied by the company BP. This is a panel that consists of 36 high-efficiency photovoltaic polycrystalline cells, providing a maximum power of $10 \mathrm{~W}$, an open circuit voltage $\left(V_{O C}\right)$ of $21 \mathrm{~V}$, and a short-circuit current $\left(I_{S C}\right)$ of $0.65 \mathrm{~A}$. Regarding the battery, that acts as the output load, it must be noted that the typical value of series resistance is $0.11 \Omega$. This, together with the panel, will establish the design specifications of the implemented power gyrator. The initial design specifications are presented in Table I.

TABLE I. - Initial design specifications.

\begin{tabular}{|c|c|}
\hline$V_{\text {in }}=20 \mathrm{~V}$ & $V_{\text {out }}=12 \mathrm{~V}$ \\
\hline$I_{\text {in }}=0,6 \mathrm{~A}$ & $I_{\text {out }}=1 \mathrm{~A}$ \\
\hline$\Delta I_{\text {out }}=5 \%$ & $\Delta V_{\text {out }}=0,5 \%$ \\
\hline & $R_{L}=0,11 \Omega$ \\
\hline
\end{tabular}

The design of the power gyrator is divided into two parts: On the one hand, the calculation of the components of the buck regulator, and, on the other, the other elements of the whole power gyrator structure, such as the filter input and the network stability.

\section{A. Component Design of the Buck Converter}

Assuming that, as design specifications, the converter operates in continuous conduction mode (CCM), that it has a nominal input voltage of $20 \mathrm{~V}$, and is desired an output voltage equal to $12 \mathrm{~V}$ and a maximum load current of $1 \mathrm{~A}$, the nominal duty cycle converter should be $60 \%$. Once obtained the duty cycle, the values of the required inductance and capacitor can be obtained, setting an output current ripple equal to $5 \%$, an output voltage ripple of $0.5 \%$, and a switching frequency equal to 50 $\mathrm{kHz}$. With these design specifications, an inductance of $1.64 \mathrm{mH}$ is used, obtaining an output current ripple of $5.85 \%$.

Finally, the standard value for the capacitor is $C=3.3 \mu \mathrm{F}$, achieving a ripple voltage equal to $0.37 \%$, which is perfectly suited to the level required for the voltage ripple at the converter output terminals.

\section{B. Component Design of the BIF Power Gyrator}

As already mentioned, a $G$-type power gyrator has a variable $g$, an LC input filter, and a stable network whose parameters should be calculated to obtain the appropriate values for the provided initial conditions. To obtain $g$, the above assumed initial conditions for currents and voltages at the converter input and output terminals are used, set $g=0.050$.
Calculating the input filter, it carries out to a standardized value of $22 \mu \mathrm{H}$ for the inductance, and a value of $1 \mu \mathrm{F}$ for the capacitor. As discussed above, G-type power gyrator structures require a stability network for ensuring their proper performance. This stability is achieved by a capacitor connected in series with a resistor, resulting in a $3.3 \mathrm{nF}$ capacitor $\left(C_{d}\right)$ and a resistance $R_{d}=2 \mathrm{k} \Omega$. These values are necessary in order to determine the stability network. In order to ensure the stability of the system, whose equations are determined by expressions (2), (3) and (4), these three expressions should be fulfilled with the obtained component values for this particular BIF Gtype gyrator.

On the other hand, the necessary control law (in this case a sliding control) for the proper operation of the $G$-type gyrator should be established. Basically, this analog controller consists of a current sensing system comprising a shunt resistance of $50 \mathrm{~m} \Omega$, a difference amplifier, a multiplier (an AD633 from Analog Devices) for the product of the input voltage established by the parameter $g$, and a comparator with some hysteresis to fix the switching frequency.

Finally, the use of a PIC microcontroller (in this case, a Microchip's PIC18F1220) achieves the tracking of the PV-panel MPP. In the case carried out in this article, the MPPT algorithm implemented has been the well-known perturb and observation (P\&O) [5]. Notice that, for this tracking, it is necessary a second current and voltage sensing, in order to measure and introduce them to the PIC microcontroller. The output of the PIC responsible for providing the value for the parameter $g$ should be an analog value; however, the PIC can only offer at its output binary states $(5 V$ or $0 V)$. To overcome this problem, an RC filter is added, in order to filter the switching frequency of the output voltage and provide an average voltage that controls the power gyrator.

\section{Implementation, Simulation and Experimental Results of the Final Implemented G-Type Gyrator Prototype}

Figure 5 shows the complete schematic of the converter BIF carried out in this work. Furthermore, Figure 6 depicts the electrical schematic of the control loop implemented for the aforementioned $G$-type gyrator, with the acquisition subsystem to provide the measures of voltages and currents for the PIC microcontroller, and the low pass filter to its output port. Finally, figure 7 shows a photograph of the complete implementation performed.

In order to corroborate the proper operation of the designed and implemented BIF G-type gyrator, it has been powered by a solar panel. Therefore, the value of its parameter $g$ is not fixed or manually adjustable, but the PIC microcontroller will be in charge of making the perturbation algorithm and monitoring to adjust the parameter $g$ in order to always assure (independently of the irradiance conditions) the maximum power of the solar panel (MPPT). The final characteristics obtained from the implemented system are: Minimum input 
voltage equal to $18 \mathrm{~V}$ and nominal $20 \mathrm{~V}$; and, output current adjustable between $1 \mathrm{~A}$ and $2.5 \mathrm{~A}$, regardless of the value of the load connected, as long as the product of the output current and the load resistance is lower than the input voltage.

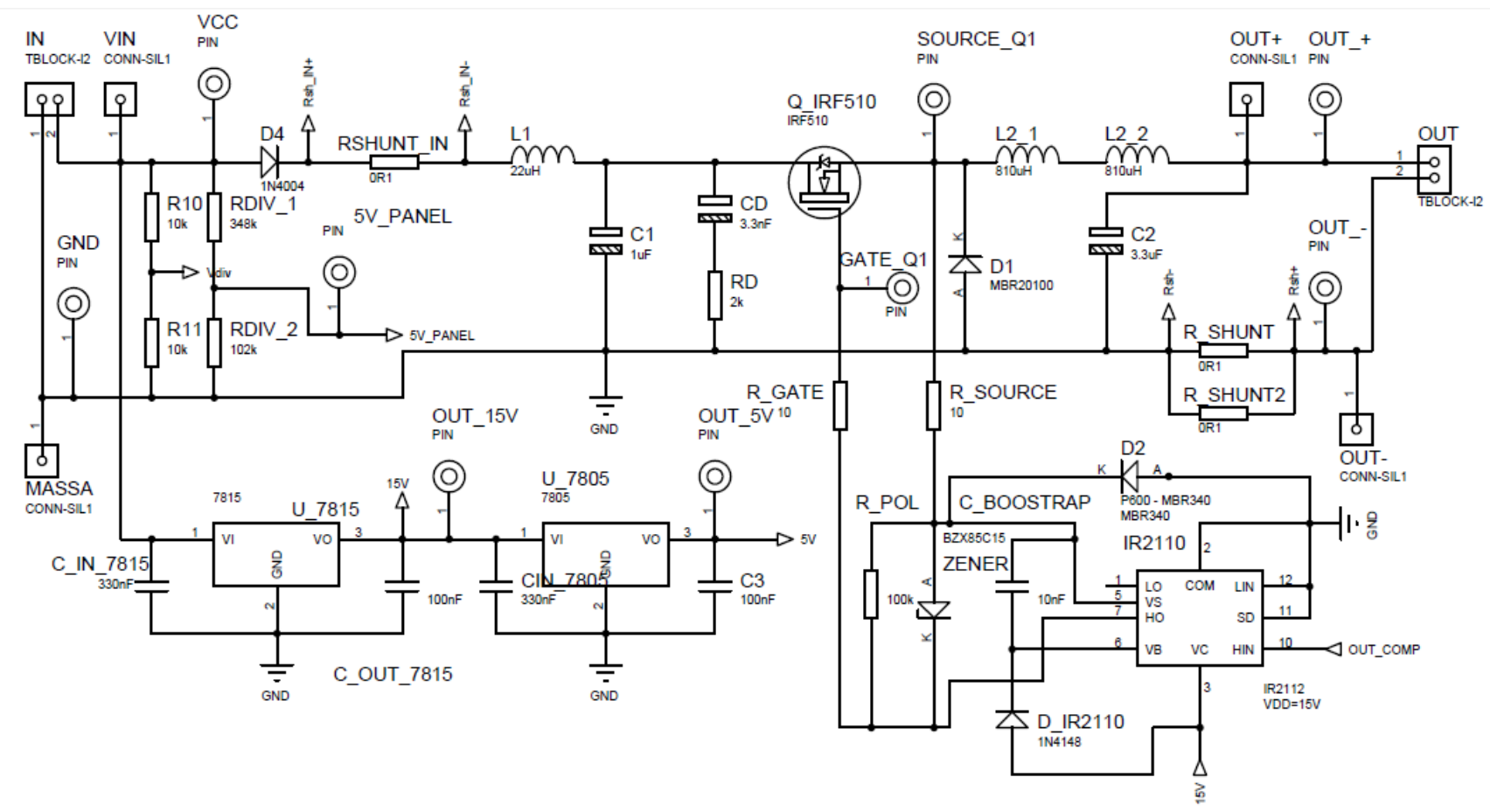

Fig. 5. Schematics of the implemented BIF G-type gyrator.

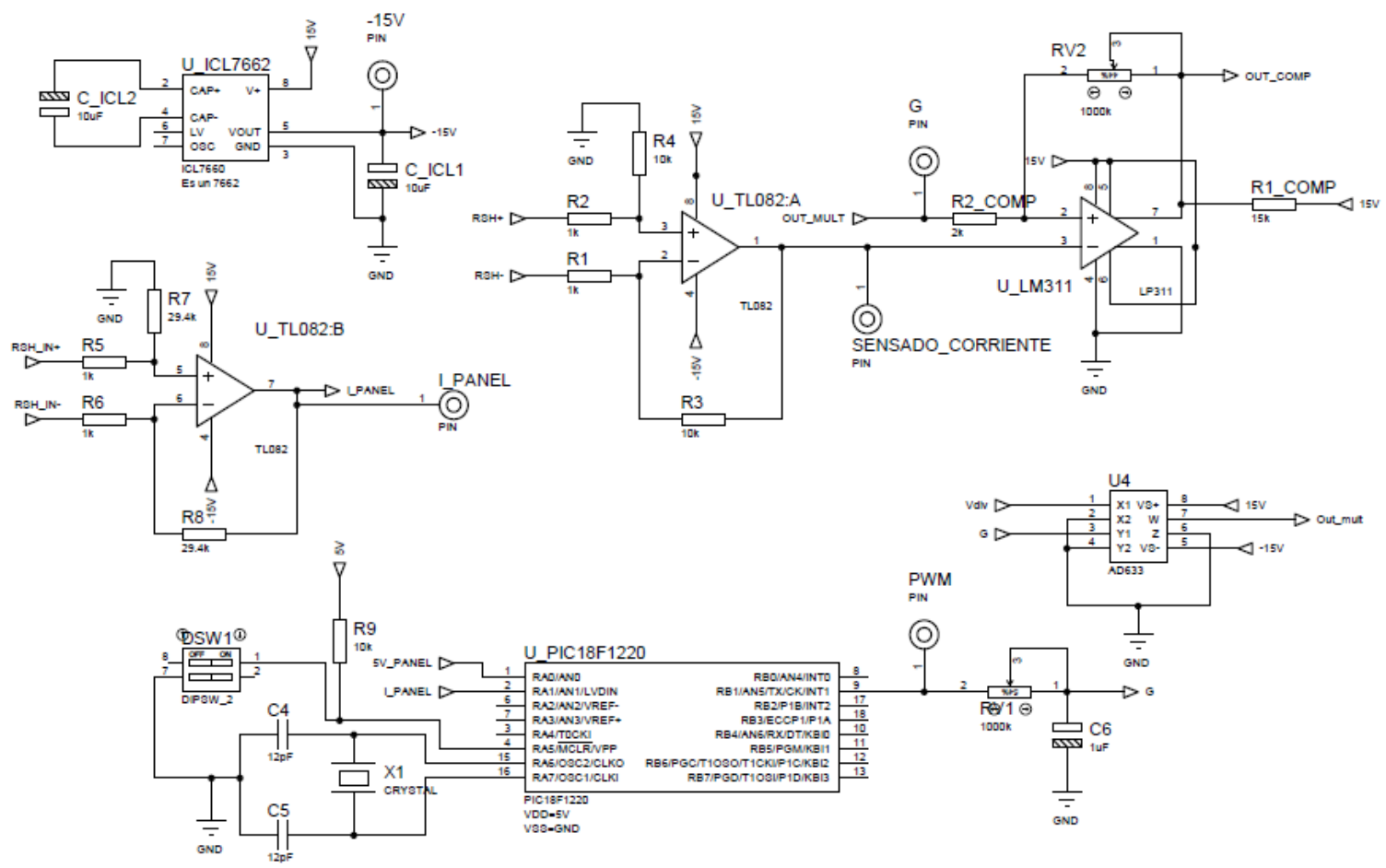

Fig. 6. Schematics of the control loop for the implemented BIF G-type gyrator.

For an input of $18 \mathrm{~V}$, a reference voltage $V_{g}$ whose value forces an input current of $1.9 A$, and an output $4.7-\Omega$ load, an output voltage and current equal to $11.8 \mathrm{~V}$ and $2.4 \mathrm{~A}$, respectively, are obtained. For the prototype of the $G$-type power gyrator held for processing power of the photovoltaic panel, different lighting conditions for the solar panel were used by means of different points of light. After verifying their behavior as G-type power gyrator, an experimental analysis of the efficiency was carried out for different values of input current (setting the value $g$ ), and maintaining the value of the load equal 
to $4.7 \Omega$ (Table II). The experimental efficiency of the $G$ type power gyrator, for different input currents, is shown in Figure 8.

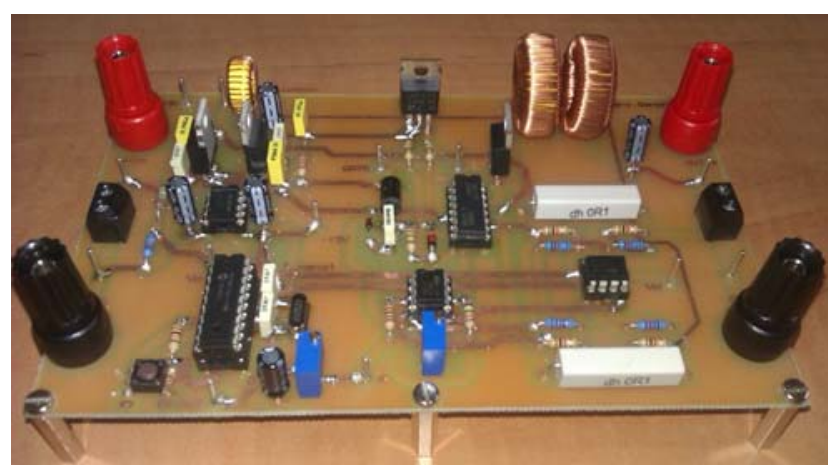

Fig. 7. Photograph of the final implemented prototype.

TABLE II. - Experimental results to obtain the efficiency of the G-type power gyrator implemented.

\begin{tabular}{|c|c|c|c|c|c|}
\hline $\boldsymbol{I}_{\text {in }}(\boldsymbol{A})$ & $\boldsymbol{V}_{\text {out }}(\mathbf{V})$ & $\boldsymbol{I}_{\text {out }}(\mathbf{A})$ & $\boldsymbol{P}_{\text {in }}(\mathbf{W})$ & $\boldsymbol{P}_{\text {out }}(\boldsymbol{W})$ & $\boldsymbol{\eta}(\mathbf{\%})$ \\
\hline 0,40 & 5,03 & 1,03 & 7,20 & 5,18 & 71,96 \\
\hline 0,50 & 5,70 & 1,17 & 9,00 & 6,67 & 74,10 \\
\hline 0,60 & 6,33 & 1,30 & 10,80 & 8,23 & 76,19 \\
\hline 0,70 & 6,92 & 1,42 & 12,60 & 9,83 & 77,99 \\
\hline 0,80 & 7,44 & 1,52 & 14,40 & 11,31 & 78,53 \\
\hline 0,90 & 7,97 & 1,63 & 16,20 & 12,99 & 80,19 \\
\hline 1,00 & 8,43 & 1,72 & 18,00 & 14,50 & 80,55 \\
\hline 1,10 & 8,85 & 1,81 & 19,80 & 16,02 & 80,90 \\
\hline 1,20 & 9,27 & 1,89 & 21,60 & 17,52 & 81,11 \\
\hline 1,30 & 9,67 & 1,97 & 23,40 & 19,05 & 81,41 \\
\hline 1,40 & 10,06 & 2,06 & 25,20 & 20,72 & 82,24 \\
\hline 1,50 & 10,43 & 2,13 & 27,00 & 22,22 & 82,28 \\
\hline 1,60 & 10,82 & 2,21 & 28,80 & 23,91 & 83,03 \\
\hline 1,70 & 11,12 & 2,27 & 30,60 & 25,24 & 82,49 \\
\hline 1,80 & 11,45 & 2,34 & 32,40 & 26,79 & 82,69 \\
\hline 1,90 & 11,78 & 2,40 & 34,20 & 28,27 & 82,67 \\
\hline 2,00 & 12,09 & 2,47 & 36,00 & 29,86 & 82,95 \\
\hline
\end{tabular}

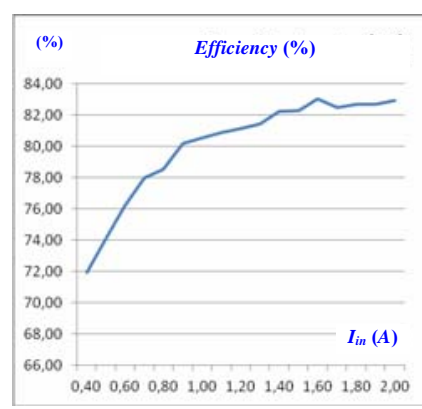

Fig. 8. Experimental efficiency of the $G$-type power gyrator for different values of the input current.

This graph shows how, as the $G$-type power gyrator works closer to the optimum point $\left(I_{i n}=2 A\right)$, the performance and efficiency are enhanced. Note that the efficiency of the gyrator is determined by the value of the load, and that, for a given output current value, different output voltages can be obtained.

\section{Conclusions}

This paper has provided, on the one hand, a classification of high efficiency switching power-gyrator structures and, on the other, the validity of their use as cells for energy processing in photovoltaic solar installations. In particular, having into account the properties of these topologies presented in the article, their inclusion in solar facilities allows increasing the performance of the whole installation.

The design, simulation and implementation of a $G$-type power gyrator are carried out throughout the article, including a sliding control implemented by means an analog controller. In addition to the use of the aforementioned switching power gyrator, a maximum power point tracking (MPPT) is mandatory in the energy processing path in order to obtain the maximum power from the photovoltaic solar panel.

Therefore, the practical implementation carried out includes a control loop of the power gyrator in order to track the aforementioned maximum power point of the photovoltaic solar panel. In the presented design, this MPPT circuit has been implemented by means of a PIC microcontroller, a Microchip's PIC18F1220, that achieves the tracking of the PV-panel MPP. In the case carried out in this article, the MPPT algorithm implemented has been the aforementioned perturb and observation ( $\mathrm{P} \& \mathrm{O})$.

\section{Acknowledgement}

This work has been partially supported by the Spanish Ministerio de Ciencia, Innovación y Universidades (MICINN)-Agencia Estatal de Investigación (AEI) and the European Regional Development Funds (FEDER), by project PGC2018-098946-B-I00.

\section{References}

[1] D. H. Tellegen, "The Gyrator, a new electric network element", Philips Research Reports, Vol. 3, pp. 81-101, Apr. 1948.

[2] S. Singer, R. W. Erickson, "Canonical Modeling of Power Processing Circuits Based on the POPI Concept", IEEE Transactions on Power Electronics, Vol. 7 (n. 1), pp. 37-43, Jan. 1992.

[3] A. Cid-Pastor, L. Martinez-Salamero, C. Alonso, J. Calvente, G. Schweitz, "Synthesis of PWM-Based Power Gyrators", Proceedings of the IEEE International Symposium on Industrial Electronics (ISIE 2005), Vol. 3, pp. 1013-1018, 20-23 Jun. 2005.

[4] A. Cid-Pastor, L. Martinez-Salamero, C. Alonso, B. Estibals, J. Alzieu, G. Schweitz, D. Shmilovitz, "Analysis and Design of Power Gyrators in Sliding-Mode Operation", Proceedings of the IEE Electric Power Applications, Vol. 152 (n. 4), pp. 821-826, 8 Jul. 2005.

[5] A. Cid-Pastor, L. Martinez-Salamero, C. Alonso, A. El Aroudi, H. Valderrama-Blavi, "Power Distribution Based on Gyrators", IEEE Transactions on Power Electronics, Vol. 24 (n. 12), pp. 2907-2909, Dec. 2009. 